\title{
Adjuvant Sunitinib for Renal Cell Carcinoma: Still at the Beginning of the Road
}

\author{
Alberto Carretero-González and Guillermo de Velasco* \\ Hospital Universitario 12 de Octubre, Madrid, Spain
}

The remarkable progress in the treatment of metastatic renal cell carcinoma (mRCC) with antiangiogenics and immunotherapy has yet to be translated to the adjuvant space, where opportunity exists for improving overall survival (OS) in patients with high risk of relapse following radical nephrectomy. In this setting, RCC trials have been hampered repeatedly by negative results $[1,2]$. Unfortunately, up to $40 \%$ of RCC patients diagnosed with locoregional disease will have a relapse after nephrectomy [3]. The S-TRAC trial has shown that sunitinib, a Vascular Endothelial Growth Factor Receptor-Tyrosine Kinase Inhibitor (VEGFR-TKI), may provide clinical benefit, improving disease-free survival (DFS) compared to placebo in RCC patients at high risk for recurrent disease [4]. This study provides the first evidence that targeting the VEGF pathway may help to delay the recurrence in RCC. However, these results are not definitive since survival data are not mature yet. Adjuvant treatments often have this feature: the purported benefit must usually be inferred long before it is experienced. If the intermediated endpoint (in this instance, DFS) is validated, then earlier and adequate decisions are possible. If not, treatment may confer risks far greater than any expected benefits.

\footnotetext{
*Correspondence to: Guillermo de Velasco, University Hospital 12 de Octubre, Avenida Córdoba S/N, 28041 Madrid, Spain. Tel.: +34 91390800; Fax: +34 914695775; E-mail: gdevelasco.gdv@gmail.com.
}

\section{SUNITINIB MET THE PRIMARY ENDPOINT}

The S-TRAC trial included patients with locoregional RCC and the study met the primary endpoint. Patients in the sunitinib group achieved a median DFS of 6.8 years (95\% CI, 5.8 to not reached) compared to 5.6 years (95\% CI, 3.8 to 6.6) in the placebo group (hazard ratio, $0.76 ; 95 \% \mathrm{CI}, 0.59$ to $0.98 ; P=0.03$ ). Was meeting this primary endpoint good enough to change practice? The worth of any trial depends on how the study was designed as well as how the results are interpreted. Certainly, the main endpoint for adjuvant treatment should be OS, unfortunately it requires long period of follow-up to show the benefit and eventually may delay available treatments for patients. Such situations have been previously seen; for instance, adjuvant trastuzumab for HER 2 + breast cancer initially showed significant improvement in DFS without an OS benefit [5].

\section{CONFLICTING RESULTS}

Concurrently, two adjuvant randomized clinical trials with VEGFR-TKIs, the PROTECT and ASSURE trials have shown negative results including patients treated with pazopanib, sorafenib and sunitinib $[6,7]$. Why the S-TRAC trial was positive? Some differences in study design may explain the conflicting results with adjuvant sunitinib, primarily 
in patient selection. The S-TRAC was the only study with central imaging review and it included the patients with the highest risk of relapse (though risk stratification was different between trials) while the ASSURE trial enrolled a broader patient population (including stage $\mathrm{T} 1 \mathrm{~b}$ or non-clear cell histology) [8, 9]. Importantly, only in the S-TRAC trial all patients were started at standard dose. Here, effects of dose and schedule on efficacy may be relevant. In fact, the PROTECT trial showed differences in outcomes between the 600- and the 800-mg pazopanib starting dose groups, with a benefit for the $800 \mathrm{mg}$ starting dose compared to placebo (HR, $0.69 ; 95 \% \mathrm{CI}, 0.51$ to $0.94 ; P=0.02$ ) [9]. Additionally, tolerability of sunitinib could had been improved, since an alternative dosing schedule of 2 weeks on- 1 week off has been shown to improve tolerability and efficacy however it was not allowed in any of the aforementioned trials [10]. It is important to comment that antiangiogenics may need longer periods of treatment to be effective, with the SORCE trial (NCT00492258) possibly answering this question.

\section{BETTER PATIENT SELECTION IS NEEDED FOR BETTER OUTCOME}

The results inform that those patients at really high risk of relapse may benefit with sunitinib. The selection of patients based on clinicopathologic factors may represent a heterogeneous group of patients. In the S-TRAC trial also included a modified very highrisk UCLA Integrated Staging System (UISS) group (tumor stage 3, no or undetermined nodal involvement, no metastasis, Fuhrman grade 2 or more, and a ECOG score of 1 or more, or tumor stage 4, local nodal involvement, or both) as a pre-planned secondary analysis, where a 2.2-year delay in DFS was seen in patients treated with sunitinib (HR, $0.74 ; P=0.04)$. Sadly, despite the improved and deep knowledge in the molecular biology of RCC, no single biomarker is guiding treatment and regrettably most trials are currently being developed without a defined robust biomarker.

\section{TOXICITY MATTERS}

Adjuvant sunitinib has been questioned due to the toxicity profile. Nevertheless, all adverse events were well-known and expected, similar to the outcomes observed in the metastatic setting. Toxic effects were manageable and treatment discontinuations were as common as in the metastatic setting [11]. The rates of serious adverse events were similar between groups and, most importantly, no deaths were attributed to toxic effects related to the study treatment. Health-related quality of life reports did not show important differences between groups, with the exception of diarrhea and loss of appetite.

Sunitinib has shown a sign for improvement in the outcomes of patients with locally advance disease. Sunitinib may be more active and increase overall survival in some patients, but it does not fit all patients. Futures studies should avoid repeat mistakes in the adjuvant setting. Adjuvant strategy needs improved molecular stratification in order to define which patients may achieve an unquestionable benefit. New and dynamic molecular tools should homogenize population in terms of recurrence risk and dependence on angiogenic pathway. Theoretically, in these groups of patients the adjuvant antiangiogenic drugs would be more valuable.

Much remains to be understood about sunitinib in the adjuvant setting but for the first time, high-risk RCC patients may have another option to be considered. Based on the S-TRAC trial, the Food and Drug Administration (FDA) has approved the use of sunitinib in the adjuvant setting [12]. An open question is whether future clinical trials and currently running clinical trials should include sunitinib as control arm. It is worth highlighting that along with neratinib and ipilimumab are the only drugs approved for adjuvant treatment for solid tumors in the last five years [13].

In conclusion, only one study with sunitinib has shown to improve DFS compared to placebo; we face many challenges but the biology of this tumor warrants the need to further explore the efficacy of antiangiogenics in selected molecular populations.

\section{REFERENCES}

[1] Clark JI, Atkins MB, Urba WJ, Creech S, Figlin RA, Dutcher JP, et al. Adjuvant high-dose bolus interleukin-2 for patients with high-risk renal cell carcinoma: A cytokine working group randomized trial. J Clin Oncol Off J Am Soc Clin Oncol 2003;21(16):3133-40.

[2] Chamie K, Donin NM, Klöpfer P, Bevan P, Fall B, Wilhelm O, et al. Adjuvant Weekly Girentuximab Following Nephrectomy for High-Risk Renal Cell Carcinoma: The ARISER Randomized Clinical Trial. JAMA Oncol 2017;3(7):913-20.

[3] Janzen NK, Kim HL, Figlin RA, Belldegrun AS. Surveillance after radical or partial nephrectomy for localized renal cell carcinoma and management of recurrent disease. Urol Clin North Am 2003;30(4):843-52. 
[4] Ravaud A, Motzer RJ, Pandha HS, George DJ, Pantuck AJ, Patel A, et al. Adjuvant Sunitinib in High-Risk Renal-Cell Carcinoma after Nephrectomy. N Engl J Med 2016;375(23):2246-54.

[5] Goldhirsch A, Gelber RD, Piccart-Gebhart MJ, de Azambuja E, Procter M, Suter TM, et al. 2 years versus 1 year of adjuvant trastuzumab for HER2-positive breast cancer (HERA): An open-label, randomised controlled trial. Lancet Lond Eng1 2013;382(9897):1021-8.

[6] Haas NB, Manola J, Uzzo RG, Flaherty KT, Wood CG, Kane $\mathrm{C}$, et al. Adjuvant sunitinib or sorafenib for high-risk, nonmetastatic renal-cell carcinoma (ECOG-ACRIN E2805): A double-blind, placebo-controlled, randomised, phase 3 trial. Lancet Lond Engl 2016;387(10032):2008-16.

[7] Motzer RJ, Haas NB, Donskov F, Gross-Goupil M, Varlamov S, Kopyltsov E, et al. Randomized Phase III Trial of Adjuvant Pazopanib Versus Placebo After Nephrectomy in Patients With Localized or Locally Advanced Renal Cell Carcinoma. J Clin Oncol Off J Am Soc Clin Oncol 2017;JCO2017735324.

[8] Zisman A, Pantuck AJ, Dorey F, Said JW, Shvarts O, Quintana D, et al. Improved prognostication of renal cell carcinoma using an integrated staging system. J Clin Oncol Off J Am Soc Clin Oncol 2001;19(6):1649-57.

[9] Patard J-J, Kim HL, Lam JS, Dorey FJ, Pantuck AJ, Zisman A, et al. Use of the University of California Los Angeles integrated staging system to predict survival in renal cell carcinoma: An international multicenter study. J Clin Oncol Off J Am Soc Clin Oncol 2004;22(16):3316-22.

[10] Guida FM, Santoni M, Conti A, Burattini L, Savini A, Zeppola $\mathrm{T}$, et al. Alternative dosing schedules for sunitinib as a treatment of patients with metastatic renal cell carcinoma. Crit Rev Oncol Hematol 2014;92(3):208-17.

[11] Motzer RJ, Hutson TE, Cella D, Reeves J, Hawkins R, Guo $\mathrm{J}$, et al. Pazopanib versus sunitinib in metastatic renal-cell carcinoma. N Engl J Med 2013;369(8):722-31.

[12] Food and Drug Administration (FDA). FDA approves sunitinib malate for adjuvant treatment of renal cell carcinoma [Internet]. Available from: https://www.fda.gov/ Drugs/InformationOnDrugs/ApprovedDrugs/ucm585686.h tm?utm_campaign=Oncology\%2011\%2F16Sun\&utm_med ium=email\&utm_source=Eloqua\&elqTrackId=b1f6f7e9d0 49432ea0be04f1df78850f\&elq=cb31f7897b4f49d6b9ab7a bff05f6883\&elqaid=1405\&elqat $=1$ \&elqCampaignId $=871$.

[13] Chan A, Delaloge S, Holmes FA, Moy B, Iwata H, Harvey VJ, et al. Neratinib after trastuzumab-based adjuvant therapy in patients with HER2-positive breast cancer (ExteNET): A multicentre, randomised, doubleblind, placebo-controlled, phase 3 trial. Lancet Oncol 2016;17(3):367-77. 